\title{
Rotor Retaining Sleeve Design for a 1.12-MW High-Speed PM Machine
}

\author{
Fengge Zhang \\ School of Electrical \\ Engineering, \\ Shenyang \\ University of \\ Technology, \\ Shenyang, 110870 , \\ China \\ zhangfg@sut.edu.cn
}

\author{
Guanghui Du \\ School of Electrical \\ Engineering, \\ Shenyang \\ University of \\ Technology, \\ Shenyang, 110870 , \\ China \\ duguanghui1104@163.com
}

\author{
Tianyu Wang \\ School of \\ Mechanical \\ Engineering, \\ Shenyang Institute \\ of Engineering, \\ Shenyang, 110136, \\ China \\ lnwangtianyu@aliyun.com
}

Guangwei Liu

Wenping Cao ${ }^{1,2}$

\author{
School of Electrical \\ Engineering, \\ Shenyang \\ University of \\ Technology, \\ Shenyang, 110870 , \\ China \\ liu-guangwei@hotmail.com
}

\author{
1 School of \\ Electronics, Electrical \\ Engineering and \\ Computer Science, \\ Queen's University \\ Belfast, Belfast, UK, \\ BT95AH \\ w.cao@qub.ac.uk \\ 2 Department of \\ Electrical Engineering \\ and Computer Science, \\ Massachusetts Institute \\ of Technology, \\ Cambridge, MA02139, \\ USA \\ wencao@mit.edu
}

\begin{abstract}
Permanent magnet synchronous machines (PMSMs) can provide excellent performance in terms of torque density, energy efficiency and controllability. However, PMs on the rotor are prone to the centrifugal force which may break their physical integrity, especially at high-speed operation. Typically, PMs are bound with carbon fiber or retained by alloy sleeves on the rotor surface. This paper is concerned with the design of a rotor retaining sleeve for a 1.12-MW 18-krpm PM machine; its electromagnetic performance is investigated by the $2 \mathrm{D}$ finite element method (FEM). Theoretical and numerical analysis of the rotor stress are carried out. For the carbon fiber protective measure, the stresses of three PM configurations and three pole filler materials are compared in terms of operating temperature, rotor speed, retaining sleeve thickness, and interference fit. Then a new hybrid protective measure is proposed and analyzed by the 2D FEM for operational speeds up to 22 krpm (1.2 times the rated). The rotor losses and machine temperatures with the carbon fiber retaining sleeve and the hybrid retaining sleeve are compared and the sleeve design is refined. Two rotors using both designs are prototyped and experimentally tested to validate the effectiveness of the developed techniques for permanent magnet machines. The developed retaining sleeve makes it possible to operate MW PM machines at high speeds of $22 \mathrm{krpm}$. This opens doors for many high-power high-speed applications such as turbo-generators, aerospace and submarine motor drives.
\end{abstract}

Index Terms - Carbon fiber, finite element method, high speed mechanical stress, operation, retaining sleeve, permanent magnet machines.

\section{INTRODUCTION}

In recent years, permanent magnet synchronous machines (PMSMs) have been widely used in safety-critical and high-performance industry due to their high power density and high efficiency. For direct-drive high-speed PMSMs, the size and weight of a motor drive

Digital Object Identifier: 10.1109/ITEC-AP.2014.6940878 system can be further and greatly reduced. They are seen in applications such as compressors, machine tools, gas turbines, flywheels, centrifuges, and vacuum pumps [1]-[5]. In the defense industry, high-speed machines are favored to provide an excellent electric drive capability for aircraft, shipboard and aircraft carriers. Especially for aircraft carriers, high-speed PM machines can significantly reduce the weight of the hull itself and thus improve payload and mobility. However, PMs cannot sustain large centrifugal forces so that a major challenge in the high-speed PMSM design is how best to retain the PMs at high-speed operations [6]-[10].

In order to keep PMs on the rotor, a high strength retaining sleeve is necessary, typically using alloy or carbon fiber [11]-[12]. In the literature, some researchers have studied [13]-[15] the properties of the alloy retaining sleeve and their influence on the rotor stress. It is found that the rotor eddy-current loss caused by the spatial harmonics and time harmonics is increased due to the use of the sleeve. This gives rise to some problems such as overheating, hotspots in the rotor and potential demagnetization of PMs. Similarly, carbon fiber is also commonly used to bind magnets and the rotor so as improve the rotor's mechanical strength [16]. It is shown in [17] that glass fiber enables safe pressing of the carbon fiber ring and to protect carbon fiber from bending at corners of the magnets. But carbon fiber has a low heat transfer coefficient, which affects the rotor's thermal performance. Therefore, a laminated semi-permeable sleeve structure from 50\% cold-worked AISI 304L stainless steel is proposed in [18] to improve the heat transfer within the rotor. So far, all the reported work is based on low-power and relatively low-speed PM motors; few have touched on MW-level PM machines or running speed of over $10 \mathrm{krpm}$. High-power high-speed applications pose a particular technical challenge to the machine design owing to their strict thermal and 
mechanical constraints. For example, the line velocity and the centrifugal force of PMs in a 1-MW 20-krpm PMSM can be in excess of $600 \mathrm{~km} / \mathrm{h}$ and $30 \mathrm{MN}$, respectively. The difficulty degree (expressed as speed $\times$ sqrt (power) in [9]) is $6.7 \times 10^{5}$ for such a machine, which is very close to the limit $\left(8 \times 10^{5}\right)$ of the surface-mount PM with sleeves. This paper addresses this challenge electromagnetically, mechanically and thermally.

Firstly, this paper investigates the electromagnetic performances of a high-speed PMSM rated 1.12 MW and $18 \mathrm{krpm}$ by the two-dimensional (2D) finite element method (FEM). Second, a numerical study of the rotor stress in a carbon fiber retaining sleeve is carried out by 2D and 3D FEM. The rotor operates up to 1.2 times the rated speed and the stresses of three PM configurations and different materials in the pole filler are studied and compared. In addition, the effect of running temperature, rotor speed, retaining sleeve thickness and interference fit (amount of interference to fit the sleeve on PMs) are investigated and optimized. Next, a new hybrid protective measure composed of Ti-alloy and carbon fiber is proposed and the rotor stresses at $20^{\circ} \mathrm{C}$ and $150^{\circ} \mathrm{C}$ are analyzed and compared to the carbon fiber sleeve at 22 $\mathrm{krpm}$. The rotor losses and machine temperature with the carbon fiber retaining sleeve and hybrid retaining sleeve are calculated and compared. Two rotors (one with a carbon fiber retaining sleeve and another hybrid retaining sleeve) are prototyped and experimentally tested at 22 $\mathrm{krpm}$ to validate the sleeve design and the electromagnetic and thermal tests are conducted for the rotor with a carbon fiber retaining sleeve at $18 \mathrm{krpm}$.

\section{Machine Structure AND EleCtromagnetic ANALYSIS}

A 1.12-MW PMSM for a pump drive application is used for analysis. Its structure is shown in Fig. 1 and its main specifications are tabulated in Table I. The PMSM has a 4-pole rotor and the PMs are mounted on the rotor surface and retained by a carbon fiber ring. PMs are made of $\mathrm{NdFeB}$ and radially magnetized. An air-water cooling system is arranged to improve the thermal transfer of the machine. The cooling air path is set in the stator slot, and a cooling liquid loop in the stator housing.

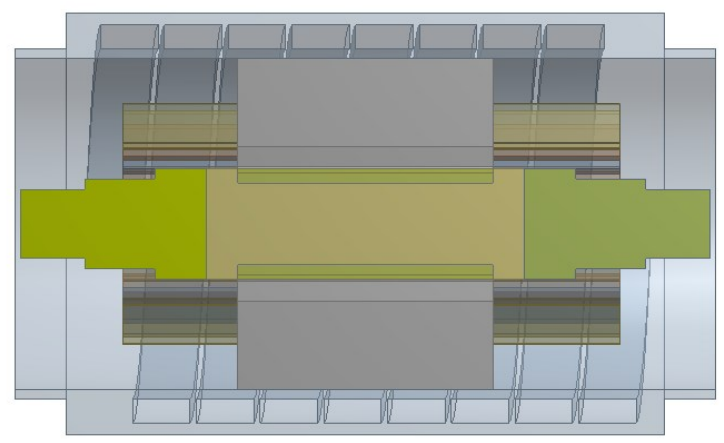

(a)

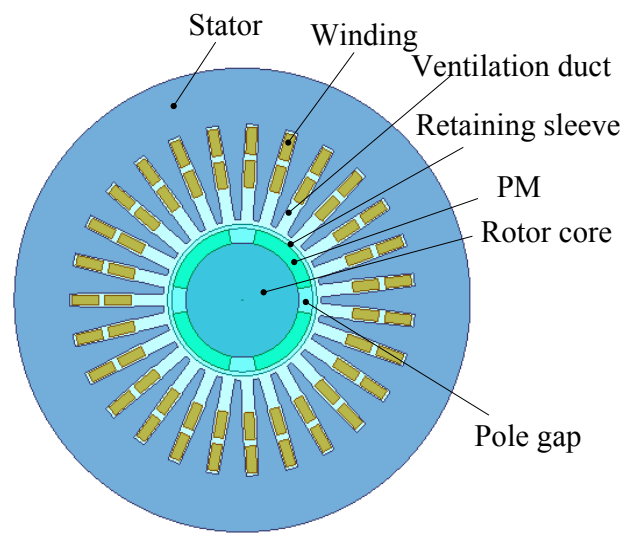

(b)

Fig. 1. (a) Axial view and (b) radial view of the PMSM.

TABLE I SPECIFICATIONS OF THE PMSM

\begin{tabular}{|l|c|}
\hline \multicolumn{1}{|c|}{ Parameter } & Value \\
\hline Rated power $(\mathrm{MW})$ & 1.12 \\
\hline Rated voltage $(\mathrm{kV})$ & 3 \\
\hline Rated speed (krpm) & 18 \\
\hline Rated current $(\mathrm{A})$ & 225 \\
\hline Rated torque $(\mathrm{Nm})$ & 594 \\
\hline Frequency $(\mathrm{Hz})$ & 600 \\
\hline Pole number & 4 \\
\hline Airgap length $(\mathrm{mm})$ & 3 \\
\hline Stator outer diameter $(\mathrm{mm})$ & 550 \\
\hline Effective stator length $(\mathrm{mm})$ & 400 \\
\hline
\end{tabular}

By using the 2D FEM in Ansoft, the electromagnetic performance of the PMSM is first analyzed at the full load condition. Fig. 2(a) shows the magnetic flux distribution of the machine and Figs. 2(b)-(d) present the electromagnetic torque, load current, and efficiency results. In Figs. 2(b), the torque has an average value of $598 \mathrm{Nm}$, which is very close to the rated value. The root mean square (rms) value of the load current in Figs. 2(c) is 224 A, which is also close to the rated value. In Figs. 2(d), the machine efficiency is generally very high with respect to the torque angle. At the rated load (load angle $=36^{\circ}$ ), the efficiency is approximately $97.6 \%$. It can be seen from the simulation results that the predicted performance of the machine has satisfied the specifications of the machine.

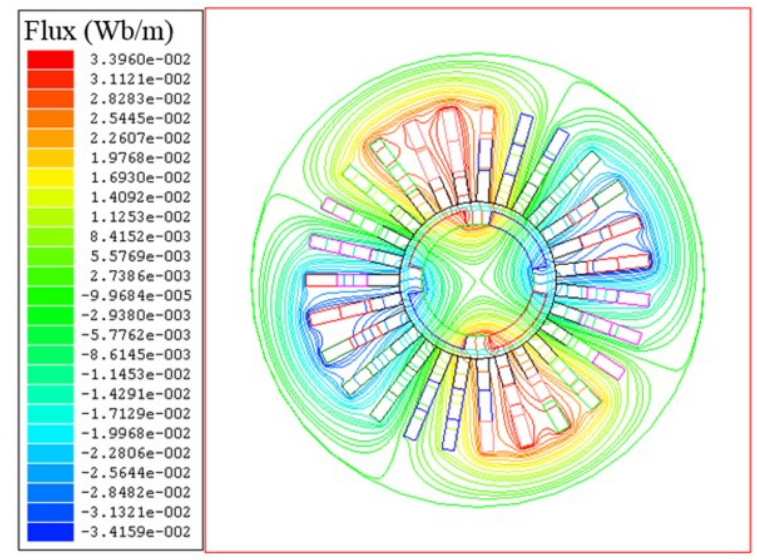

(a) 


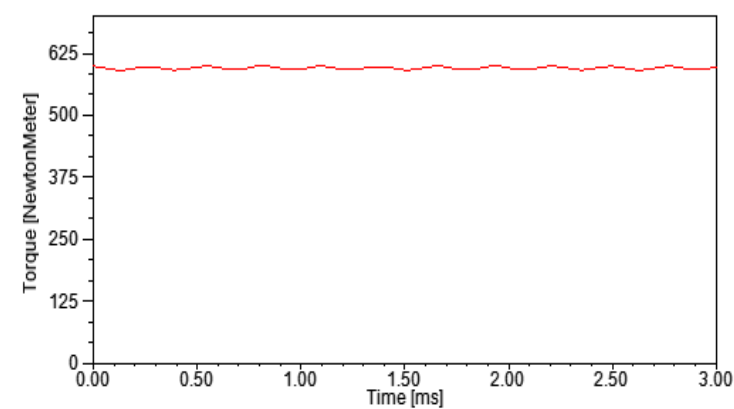

(b)

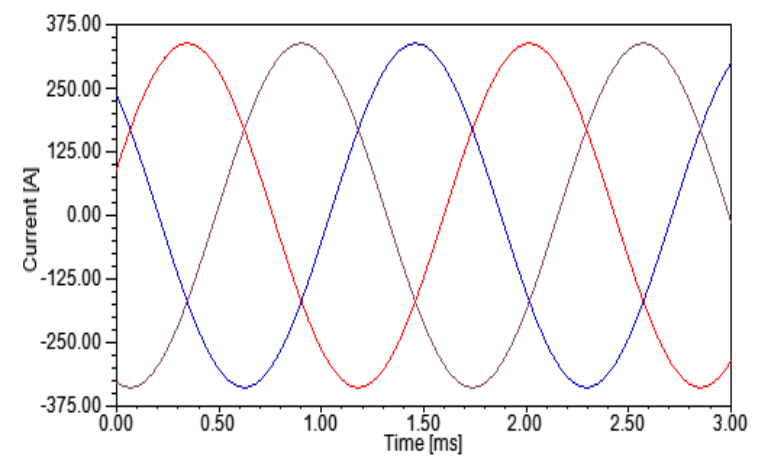

(c)

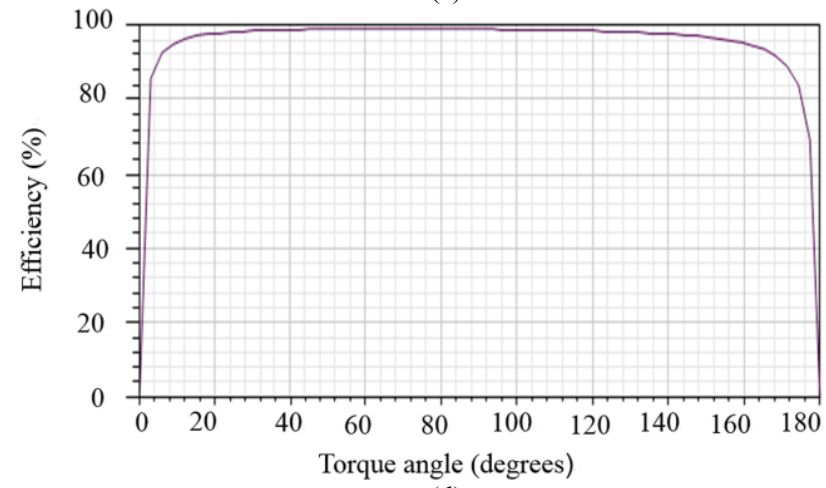

(d)

Fig. 2 Electromagnetic performance of the PMSM. (a) Magnetic flux distribution, (b) torque at the full load condition, (c) the current at the full load condition, (d) efficiency versus torque angle.

\section{ROTOR STRESS ANALYSIS}

In practice, PMSMs utilize either sintered $\mathrm{NdFeB}$ or SmCo for high-performance applications. However, their maximum permissible tension is about $120 \mathrm{MPa}$ and 30 $\mathrm{MPa}$, respectively. $\mathrm{NdFeB}$ is used in the proposed PMSM because of its higher mechanical rigidity. In general, all PMs are prone to large centrifugal forces and thus some protection measures should be adopted for high-speed operations. There are commonly two methods used: one is to bind PMs with either glass or carbon fiber, the other is to retain PMs with a high-strength retaining sleeve made of metal alloy. The material properties of typical retaining sleeves and PMs are obtained from the material manufacturers and shown in Table II. Although the maximum permissible tensile stress of PMs is $120 \mathrm{MPa}$, their permissible compression stress is $1100 \mathrm{MPa}$. Considering the bending effect, the maximum stress of carbon fiber is $1400 \mathrm{MPa}$ in the tangential direction and $-100 \mathrm{MPa}$ in the radial direction, respectively. The glass-fiber bandage is not strong enough to hold magnets in place if the circumferential speed is over $150 \mathrm{~m} / \mathrm{s}$ [16]. In this case, it is about $180 \mathrm{~m} / \mathrm{s}$ for the proposed PMSM so that a retaining sleeve is needed. As a result, the titanium alloy retaining sleeve and carbon fiber retaining sleeve are better options but the former induces an additional eddy current loss. This paper analyzes two types of the retaining sleeve structure: a carbon fiber retaining sleeve and a hybrid titanium alloy-carbon fiber retaining sleeve. The thickness of the retaining sleeve and rotor stress must be accurately calculated to balance out the stress. Otherwise, the excessive stress might cause a serious damage to the rotor, as shown in [16].

\begin{tabular}{|c|c|c|c|c|c|}
\hline \multirow{2}{*}{ Material property } & \multirow{2}{*}{$\begin{array}{c}\mathrm{PM} \\
(\mathrm{NdFeB}) \\
\end{array}$} & \multirow{2}{*}{\begin{tabular}{|c} 
Ti-Alloy \\
{$[11]$}
\end{tabular}} & \multicolumn{2}{|c|}{ Carbon Fiber } & \multirow{2}{*}{ Plastics } \\
\hline & & & Tangential & Radial & \\
\hline Density $\left(\mathrm{kg} / \mathrm{m}^{3}\right)$ & 7400 & 7850 & \multicolumn{2}{|c|}{1620} & 1.3 \\
\hline Elastic modulus (GPa) & 160 & 206 & 140 & 8.8 & 12 \\
\hline Poisson's ratio & 0.24 & 0.31 & 0.28 & 0.015 & 0.34 \\
\hline $\mathrm{CTE}\left(\mu \mathrm{m} / \mathrm{m} /{ }^{\circ} \mathrm{C}\right)$ & 8 & 13.25 & $-1[17]$ & 35 & 32 \\
\hline $\begin{array}{c}\text { Maximum permissible } \\
\text { stress }(\mathrm{MPa})\end{array}$ & \begin{tabular}{|c|} 
tensile stress \\
120 \\
compressive \\
stress 1100 \\
\end{tabular} & 1100 & $1400[17]$ & $-100[17]$ & 180 \\
\hline $\begin{array}{c}\text { Electrical conductivity } \\
(\mathrm{S} / \mathrm{m})\end{array}$ & 629000 & 610000 & 300 & & 0 \\
\hline $\begin{array}{c}\text { Thermal conductivity } \\
(\mathrm{W} /(\mathrm{m} \cdot \mathrm{K}))\end{array}$ & 12 & 16 & 0.7 & & 1.2 \\
\hline
\end{tabular}

\section{A. Theoretical Analysis of Rotor Stress}

Plane stress and plane strain models are commonly used for the stress analysis. In literature, it is claimed that the plain stress model is better suited for disks while the plain strain model is for slender cylinders [17], depending on the ratio of the axial length to the outer diameter. However, an extensive study using both models for a slender cylinder and a disk carbon fiber rotor has proved that the analytical results between the two models are almost identical. In this study, we initially tried both models in FEM and numerical results also confirmed the conclusion from [19] but the implementation of the former in FEM is much easier than the latter. Therefore, the plane stress model is adopted in the following analysis. A simplified plane model is shown in Fig. 3.

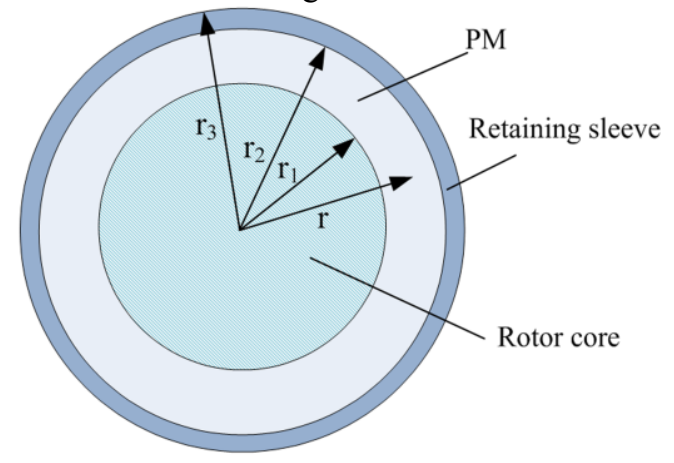

Fig. 3. The stress model.

In a cylindrical system, the relationship between the strain and stress can be found by: 


$$
\left[\begin{array}{c}
\varepsilon_{\mathrm{r}} \\
\varepsilon_{\theta}
\end{array}\right]=\left[\begin{array}{cc}
\frac{1}{E_{\mathrm{r}}} & -\frac{v_{\mathrm{r} \theta}}{E_{\theta}} \\
-\frac{v_{\theta \mathrm{r}}}{E_{\mathrm{r}}} & \frac{1}{E_{\theta}}
\end{array}\right]\left[\begin{array}{l}
\sigma_{\mathrm{r}} \\
\sigma_{\theta}
\end{array}\right]
$$

where $\varepsilon_{\mathrm{r}}$ and $\varepsilon \theta$ denote the strain, $\sigma_{\mathrm{r}}$ and $\sigma_{\theta}$ denote the stress, $r$ and $\theta$ correspond to the radial and tangential direction, respectively. $E_{\mathrm{r}}$ and $E_{\theta}$ are the radial and tangential modulus of elasticity, respectively. $v_{\theta_{\mathrm{r}}}$ and $v_{\mathrm{r} \theta}$ are the material poisson ratios in $r$ and $\theta$ directions, respectively.

If the axial strain is neglected, the balance equation can be expressed as

$$
\frac{d \sigma_{\mathrm{r}}}{d r}+\frac{\sigma_{\mathrm{r}}-\sigma_{\theta}}{r}+\rho \omega^{2} r=0
$$

where $\omega$ is the angular speed of the machine and $\rho$ is the material density.

$$
\varepsilon_{\theta}=\frac{u_{\mathrm{r}}}{r}, \varepsilon_{\mathrm{r}}=\frac{\partial u_{\mathrm{r}}}{\partial r}
$$

By combining (1)-(3), the radial displacement $u_{\mathrm{r}}$ can be obtained:

$$
E_{\mathrm{r}} r^{2} \frac{d^{2} u_{\mathrm{r}}}{d r^{2}}+E_{\mathrm{r}} r \frac{d u_{\mathrm{r}}}{d r}-E_{\theta} u_{\mathrm{r}}=-\rho \omega^{2} r^{3}\left(1-v_{\theta \mathrm{r}} v_{\mathrm{r} \theta}\right)
$$

If $K=\sqrt{\frac{E_{\theta}}{E_{\mathrm{r}}}}$, the radial displacement and stress of the sleeve can be given by

$$
\left\{\begin{aligned}
u_{\mathrm{r} 1}= & A r^{k}+B r^{-k}-\frac{\left(1-v_{\theta \mathrm{r}} v_{\mathrm{r} \theta}\right)}{9 E_{\mathrm{r}}-E_{\theta}} \rho \omega^{2} r^{3} \\
\sigma_{\mathrm{r} 1}= & \frac{E_{\mathrm{r}} K^{2} r^{k-1}\left(A-B r^{-2 k}\right)+E_{\mathrm{r}} v_{\theta \mathrm{r}} r^{k-1}\left(A+B r^{-2 k}\right)}{1-v_{\theta \mathrm{r}} v_{\mathrm{r} \theta}} \\
& -\frac{\left(3+v_{\theta \mathrm{r}}\right)}{9-K^{2}} \rho \omega^{2} r^{2} \\
\sigma_{\theta 1}= & \frac{E_{\theta} r^{k-1}\left(A+r^{-2 k}\right)+E_{\theta} v_{\mathrm{r} \theta} r^{k-1}\left(A+B r^{-2 k}\right)}{1-v_{\theta \mathrm{r}} v_{\mathrm{r} \theta}} \\
& -\frac{\left(3+v_{\mathrm{r} \theta}\right)}{9-K^{2}} \rho \omega^{2} r^{2}
\end{aligned}\right.
$$

where $A$ and $B$ are the boundary coefficients, $u_{\mathrm{r} 1}$ is the sleeve radial displacement, $\sigma_{\mathrm{r} 1}$ and $\sigma_{\theta 1}$ are the radial stress and tangential stress of the sleeve, respectively.

The interference fit effect between PM and sleeve must be considered. Given that the PM is an isotropic material,

$$
\left\{\begin{array}{l}
E_{r}=E_{\theta}=E_{\mathrm{m}} \\
v_{r \theta}=v_{\theta r}=v_{\mathrm{m}}
\end{array}\right.
$$

After combining (6) and (4), the PM's radial displacement can be obtained

$$
\begin{gathered}
E_{m} r^{2} \frac{d^{2} u_{r 2}}{d r^{2}}+E_{m} r \frac{d u_{r 2}}{d r}-E_{m} u_{r 2}=-\rho_{m} \omega^{2} r^{3}\left(1-v_{m}^{2}\right) \\
u_{r 2}=C r+\frac{D}{r}-\frac{\left(1-v_{m}{ }^{2}\right)}{8 E_{m}} \rho \omega^{2} r^{3}
\end{gathered}
$$

The PM radial stress $\sigma_{\mathrm{r} 2}$ and $\mathrm{PM}$ tangential stress $\sigma_{\theta 2}$ are:

$$
\left\{\begin{aligned}
\sigma_{r 2}= & \frac{E_{\mathrm{m}}}{1-v_{\mathrm{m}}^{2}}\left[\frac{-\left(3+v_{\mathrm{m}}\right)\left(1-v_{\mathrm{m}}^{2}\right) \rho_{\mathrm{m}} \omega^{2} r^{2}}{8 E_{\mathrm{m}}}+\right. \\
& \left.\left(1+v_{\mathrm{m}}\right) C-\left(1-v_{\mathrm{m}}\right) D / r_{2}\right] \\
\sigma_{\theta 2}= & \frac{E_{\mathrm{m}}}{1-v_{\mathrm{m}}^{2}}\left[\frac{-\left(1+3 v_{\mathrm{m}}\right)\left(1-v_{\mathrm{m}}^{2}\right) \rho_{\mathrm{m}} \omega^{2} r^{2}}{8 E_{\mathrm{m}}}+\right. \\
& \left.\left(1+v_{\mathrm{m}}\right) C-\left(1-v_{\mathrm{m}}\right) D / r_{2}\right]
\end{aligned}\right.
$$

where $C$ and $D$ are the boundary coefficients, $\rho_{\mathrm{m}}$ is the density of PMs.

In this machine, the rotor core exerts stress on the PMs, the rotor core radial displacement $u_{\mathrm{r} 3}$, the rotor core radial stress $\sigma_{\mathrm{r} 3}$ and the rotor core tangential stress $\sigma_{\theta 3}$ can be found by Eqs (8) and (9), based on the elastic modulus and the poisson ratio of the rotor core. In this case, there are six boundary coefficients [17]:

$$
\left\{\begin{array}{l}
\sigma_{\mathrm{r} 3}(0) \neq \infty \\
\sigma_{\mathrm{r} 2}\left(r=r_{1}, \omega\right)-\sigma_{\mathrm{r} 1}\left(r=r_{1}, \omega\right)=0 \\
\sigma_{\mathrm{r}}\left(r=r_{2}, \omega\right)-\sigma_{\mathrm{r} 2}\left(r=r_{2}, \omega\right)=0 \\
u_{\mathrm{r} 2}\left(r_{2}\right)-u_{\mathrm{r}}\left(r_{2}\right)=\delta \\
u_{\mathrm{r} 2}\left(r_{1}\right)-u_{\mathrm{r} 3}\left(r_{1}\right)=0 \\
\sigma_{\mathrm{r}}\left(r=r_{3}, \omega\right)=0
\end{array}\right.
$$

When the machine operates at high temperature, the thermal expansion will be an issue which needs to be taken into account. The relationship between strain and stress can be found by:

$$
\left[\begin{array}{c}
\varepsilon_{\mathrm{r}} \\
\varepsilon_{\theta}
\end{array}\right]=\left[\begin{array}{cc}
\frac{1}{E_{\mathrm{r}}} & -\frac{v_{\mathrm{r} \theta}}{E_{\theta}} \\
-\frac{v_{\theta \mathrm{r}}}{E_{\mathrm{r}}} & \frac{1}{E_{\theta}}
\end{array}\right]\left[\begin{array}{l}
\sigma_{\mathrm{r}} \\
\sigma_{\theta}
\end{array}\right]+\left[\begin{array}{l}
\alpha_{r} \\
\alpha_{\theta}
\end{array}\right] \Delta T
$$

where $\alpha_{\mathrm{r}}$ and $\alpha_{\theta}$ denote the radial and tangential CTE, respectively, and $\Delta T$ is the temperature difference between the machine and the ambient.

The equation for radial displacement can be expressed as [17]:

$$
\frac{d^{2} u_{r}}{d r^{2}}+\frac{1}{r} \frac{d u_{r}}{d r}-h \frac{u_{r}}{r^{2}}+\frac{P}{r} \Delta T+Q \rho \omega^{2} r=0
$$

where $h, P$ and $Q$ are the constants dependent on material properties. The radial displacement, radial stress and tangential stress of rotor can be obtained in the same way as Eqs. (3)-(10).

In order to ensure the safe operation of the rotor at high speeds, the stress of the sleeve and PMs running at $120 \%$ rated speed and any temperature must be within the material stress threshold.

$$
\left\{\begin{array}{l}
\sigma_{r 1}<\sigma_{r s} \\
\sigma_{\theta 1}<\sigma_{\theta s} \\
\sigma_{r 2}<\sigma_{c n} \\
\sigma_{\theta 2}<\sigma_{t m}
\end{array}\right.
$$

where $\sigma_{\mathrm{r} 1}$ and $\sigma_{\mathrm{r} 2}$ are the radial stress of retaining sleeve and PMs, respectively; $\sigma_{\theta_{1}}$ and $\sigma_{\theta_{2}}$ are the tangential stress of retaining sleeve and PMs, respectively; $\sigma_{r s}$ and $\sigma_{\theta}$ are the maximum permissible radial stress and tangential stress of carbon fiber; $\sigma_{t m}$ is the maximum permissible compressive stress of PMs; $\sigma_{c m}$ is the maximum 
permissible tensile stress of PM. The radial stress of PMs is mainly compressive stress at high-speed rotation due to the constraints of the rotor core and sleeve but the tangential stress of PMs is mainly tension at high-speed rotation.

\section{B. Numerical Stress Models}

A numerical model with no pole gaps between PMs is built in $2 \mathrm{D}$ and $3 \mathrm{D}$ to investigate the stress of the high-speed PMSM. Fig. 4 shows the stress results taking the radially outward direction as a positive direction for radial stress and the rotational direction as a positive direction for tangential stress. Table III presents the comparison of the rotor stress at the operating temperature of $150^{\circ} \mathrm{C}$ and the rotation speed of $22 \mathrm{krpm}$. As can be seen from these, the stress results from the $2 \mathrm{D}$ and $3 \mathrm{D}$ FE models have a reasonable agreement with the analytical results.

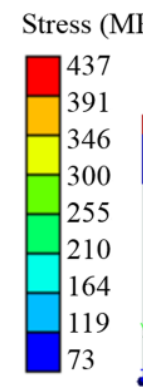

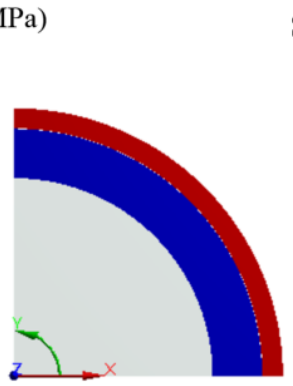

(a)

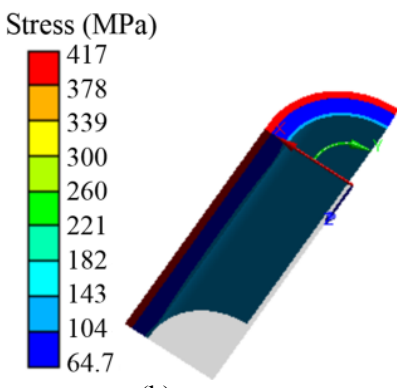

(b)
Fig. 4. Stress models at $150^{\circ} \mathrm{C}$ and $22 \mathrm{krpm}$. (a) $2 \mathrm{D}$ model. (b) $3 \mathrm{D}$ model

TABLE III

COMPARISON OF ROTOR STRESS RESULTS AT $150^{\circ} \mathrm{C}$

\begin{tabular}{|l|c|c|c|}
\hline \multicolumn{1}{|c|}{ Stress (MPa) } & Analytical & $2 \mathrm{D}$ & $3 \mathrm{D}$ \\
\hline Sleeve: inner, radial & -22 & -32 & -28 \\
\hline Sleeve: inner, tangential & 423 & 437 & 417 \\
\hline PM: inner, radial & -24 & -31 & -16 \\
\hline PM: inner, tangential & 122 & 114 & 110 \\
\hline
\end{tabular}

\section{Numerical Analysis of Rotor Stress}

In order to gain a better understanding of the rotor stress, three PM configurations $(\mathrm{A}, \mathrm{B}, \mathrm{C})$ are considered in the proposed PMSM, as shown in Fig. 5. These are:

A) PMs are not segmented and pole gaps are not filled.

B) PMs in each pole are divided into three segments and pole gaps are not filled.

C) PMs in each pole are divided into three segments and pole gaps are filled with pole filler (high temperature and high intensity non-magnetic plastics, whose properties is shown in Table II).

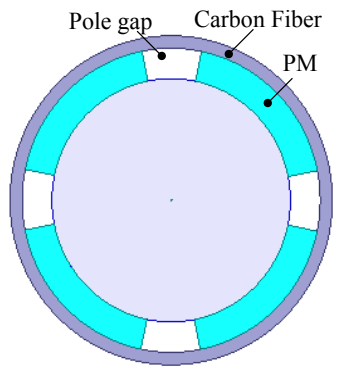

(a)

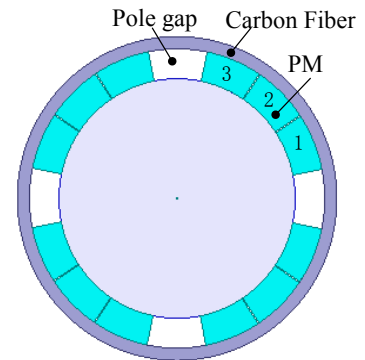

(b)

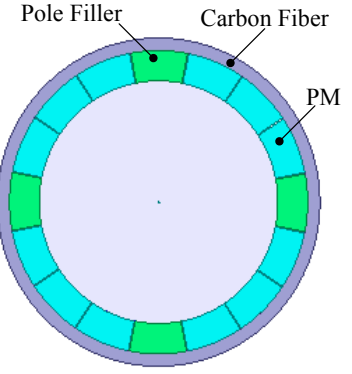

(c)

Fig. 5. Three PM configurations. (a) A, (b) B, (c) C.

For three configurations, the thickness of carbon fiber is $7 \mathrm{~mm}$ and the interference fit between the carbon fiber and the PMs is $0.12 \mathrm{~mm}$. In configurations $\mathrm{B}$ and $\mathrm{C}$, three segments PMs in each pole are bonded by the adhesive. The carbon fiber is wrapped around the rotor by specialized companies. Considering the speed of $22 \mathrm{krpm}$ and the operating temperature of $150^{\circ} \mathrm{C}$, the rotor stress is calculated in Ansys Workbench and shown in Figs. 6-8. It can be seen that there is little difference in the rotor stress between configurations $\mathrm{A}$ and $\mathrm{B}$, suggesting that the number of PM segments has little impact on the rotor stress. The maximum tangential stress of the sleeve for configuration $\mathrm{A}$ is approximately $676 \mathrm{MPa}$, which is concentrated at the pole gap of the sleeve. From Fig. 8, the maximum tangential stress and radial stress of the carbon fiber retaining sleeve in configuration $\mathrm{C}$ is $488 \mathrm{MPa}$ and $-81 \mathrm{MPa}$, respectively, which are much lower than these for configurations $\mathrm{A}$ and $\mathrm{B}$. The radial stress between the $\mathrm{PMs}$ and rotor core in configuration $\mathrm{C}$ is higher than these for configurations $\mathrm{A}$ and $\mathrm{B}$ because of significant expansion of plastics at high temperatures. However, there is very little change in the PMs' tangential stress among the three configurations. The radial stresses of PMs in the three configurations are the compressive stress and they are much lower than the maximum permissible compressive stress of PMs. The tangential stress of PMs is the tensile stress and it is also lower than the maximum permissible tension of PMs. It is effective to reduce the sleeve stress and improve the reliability of the rotor by filling the pole gap with pole fillers.

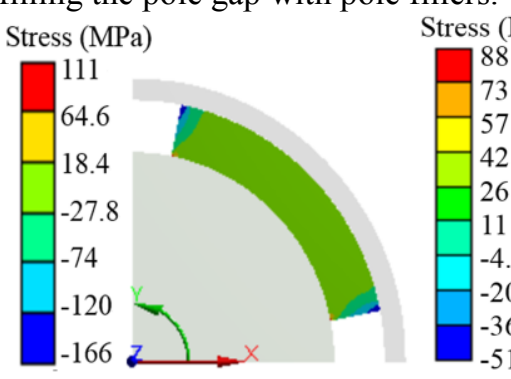

(a)

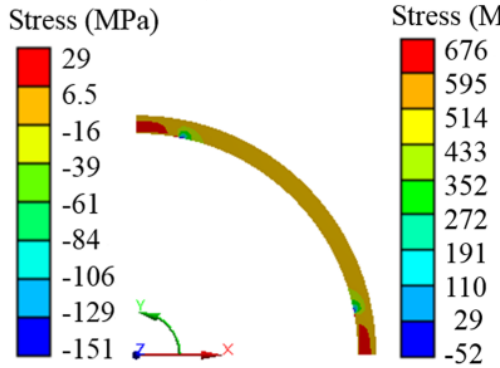

(c)

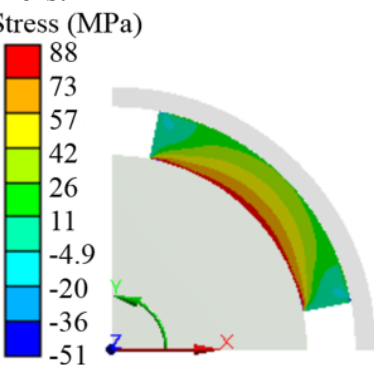

(b)

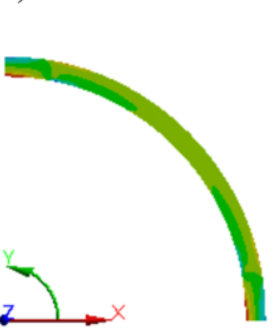

(d) 
Fig. 6. Stress distribution for configuration A. (a) Radial stress of PMs. (b) Tangential stress of PMs. (c) Radial stress of the carbon fiber sleeve. (d) Tangential stress of the carbon fiber sleeve.

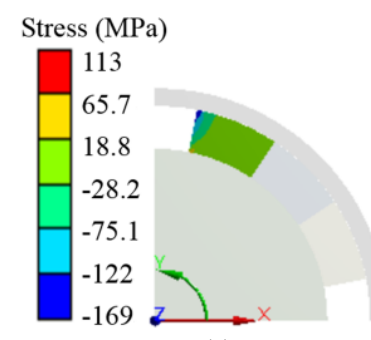

(a)

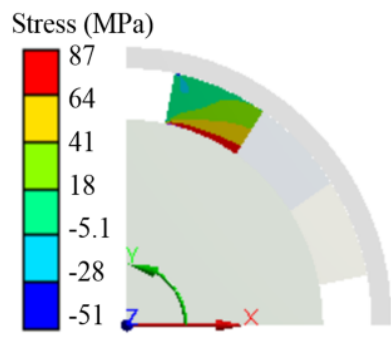

(b)

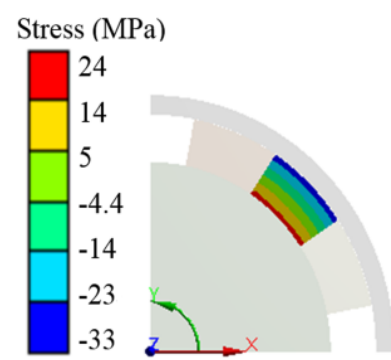

(c)

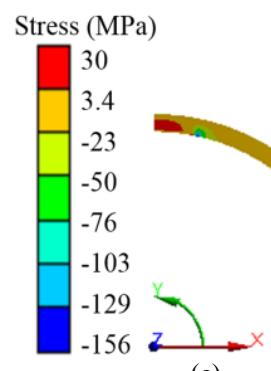

(e)

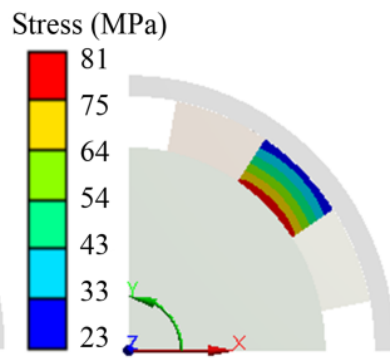

(d)

Fig. 7. Stress distribution for configuration B. (a) Radial stress of PM segment 3, (b) Tangential stress of PM segment 3, (c) Radial stress of PM segment 2, (d) Tangential stress of PM segment 2, (e) Radial stress of the carbon fiber sleeve. (f) Tangential stress of the carbon fiber sleeve.

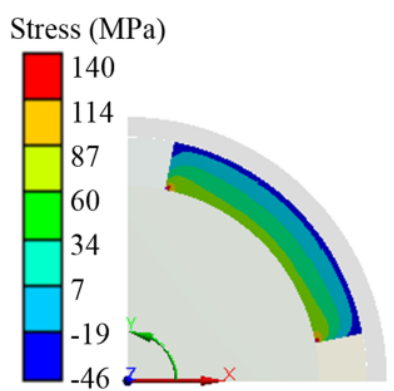

(a)

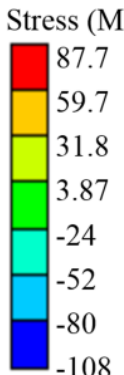

$-108$

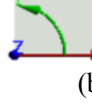

(b)

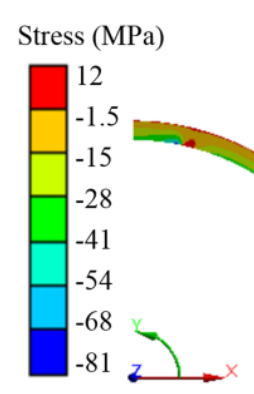

(c)
Stress (MPa)

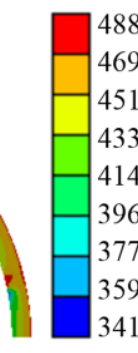

341

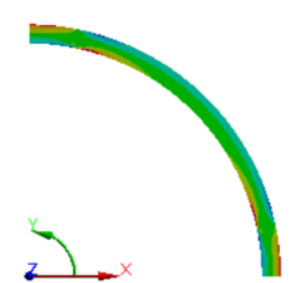

(d)
Fig. 8. Stress distribution for configuration C. (a) Radial stress of PMs,

(b) Tangential stress of PMs, (c) Radial stress of the carbon fiber sleeve.

(d) Tangential stress of the carbon fiber sleeve.

\section{Effect of Different Pole Filler Materials}

There are three materials available for the pole filler: plastics, carbon fiber, and Ti-alloy. Again, the rotor stresses using the three materials as the pole filler are calculated by the $2 \mathrm{D}$ FEM at $22 \mathrm{krpm}$ and $150^{\circ} \mathrm{C}$. As shown in Fig. 9, because of higher CET of plastics than others, the PM's radial stress and the sleeve's radial and tangential stress are at maximum with the plastic filler while they are at minimum with the Ti-alloy filler. The PM's tangential stress peaks with the Ti-alloy filler. But the Ti-alloy has a higher electrical conductivity, giving rise to eddy current loss. The rotor stress with the carbon fiber filler lies in the middle of the three filler materials, but carbon fiber is more difficult and costly to apply to the pole filler.

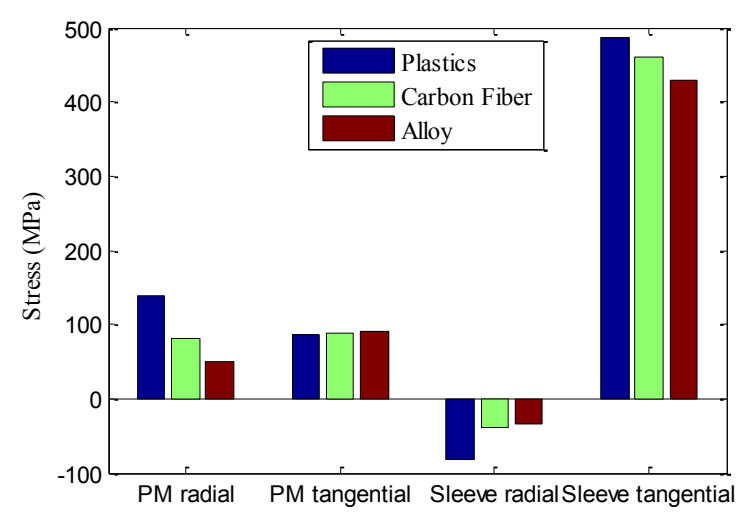

Fig. 9. The rotor stress for different materials used as the pole filler.

\section{E. Effect of Operating Temperature and Speed}

In theory, the rotor stress is also influenced by the operating temperature and the rotational speed. Compared with the radial stress, the tangential stresses of PMs and carbon fiber retaining sleeve might be more excessive. Therefore, the tangential stresses of PMs and the sleeve versus the temperature and speed are stuided based on configuration $\mathrm{C}$ and the results are shown in Fig. 10. In this simulation study, the speed changes from 0 to 30 $\mathrm{krpm}$ and the running temperature changes from 20 to $150^{\circ} \mathrm{C}$. In Fig. 10 , the stresses at all conditions are maximum. The tangential stress of PMs is the highest at $30 \mathrm{krpm}$ and $150^{\circ} \mathrm{C}$. The tangential stress of PMs increase significantly with both the speed and running temperature. The tangential stress of the carbon fiber sleeve increases significantly with the running temperature but slightly with the speed. When the speed is $22 \mathrm{krpm}(120 \%$ of the rated speed) and the operating temperature is $150^{\circ} \mathrm{C}$, the tangential stress of the carbon fiber sleeve is much higher than the rotor stress at $22 \mathrm{krpm}$ and $20^{\circ} \mathrm{C}$. 


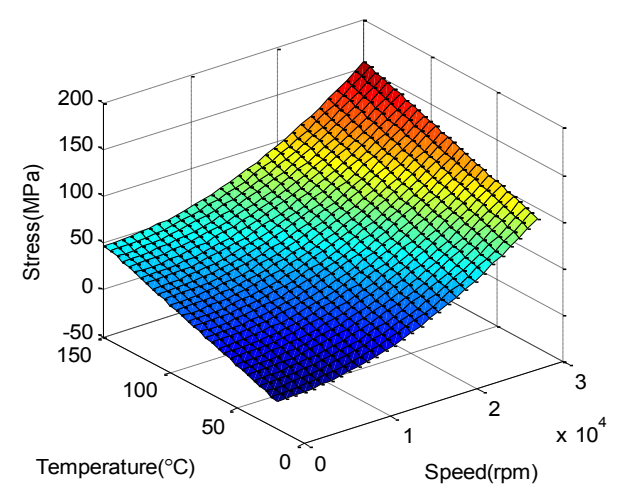

(a)

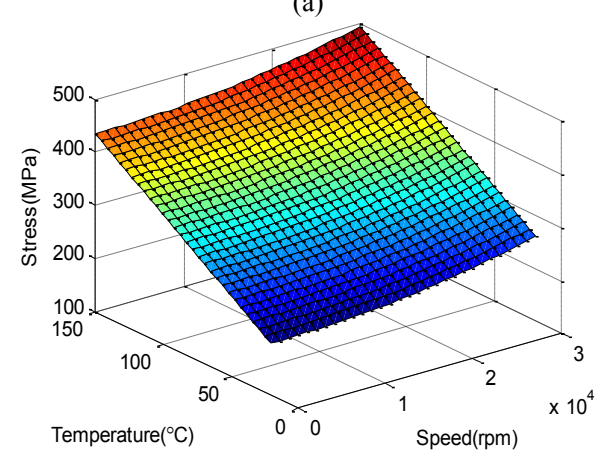

(b)

Fig. 10. The stress versus temperature and speed. (a) Tangential stress of PMs. (b) Tangential stress of the carbon fiber sleeve.

\section{F. Effect of the Sleeve Thickness and Interference Fit}

For the carbon fiber sleeve, an interference fitting is generally used in the rotor assembly to bind the PMs which can result in extra rotor stress. Based on configuration $\mathrm{C}$, the sleeve thickness is changed from 1 to $10 \mathrm{~mm}$ and the interference fit from 0 to $0.2 \mathrm{~mm}$. The simulation results for $22 \mathrm{krpm}$ and $20^{\circ} \mathrm{C}$ conditions are presented in Fig. 11. The tangential stress of PMs gradually decreases with the interference fit and change little with the sleeve thickness. The tangential stress of PMs is the lowest at the $0.2-\mathrm{mm}$ interference fit and the 10-mm sleeve thickness. However, the tangential stress of the carbon fiber sleeve increases with the interference fit and decreases with the sleeve thickness while it peaks at the $0.2-\mathrm{mm}$ interference fit and the $1-\mathrm{mm}$ sleeve thickness. It is helpful to mitigate the PM and sleeve stress with a higher sleeve thickness but the resultant need for more PMs to generate required airgap flux density gives rise to heat dissipation issues. Similarly, the increased interference fit is helpful to mitigate PM tangential stress but it would increase sleeve tangential stress. Therefore, it needs to strike a balance between the sleeve thickness and the interference fit.

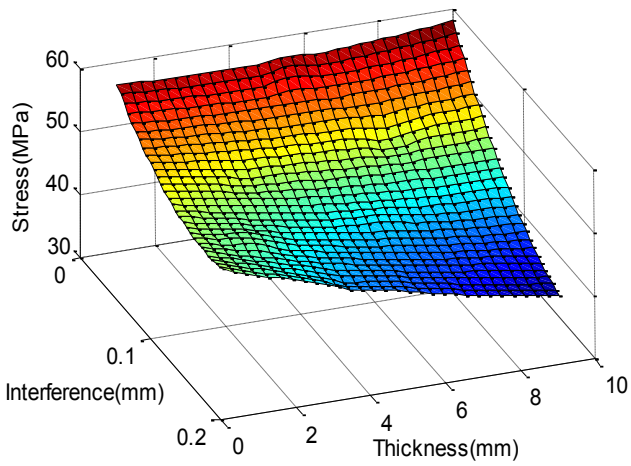

(a)

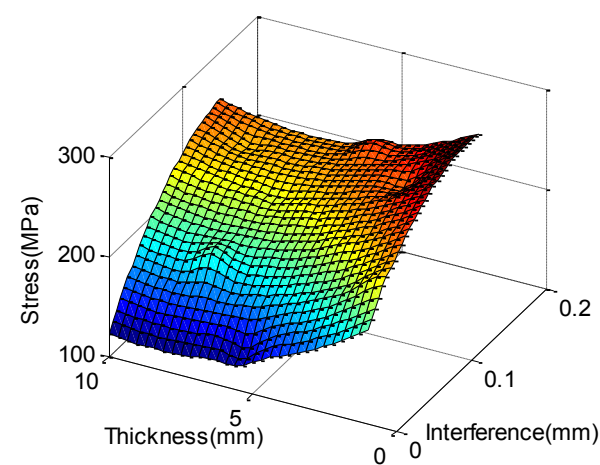

(b)

Fig. 11. The stress versus the sleeve thickness and the interference fit. (a) Tangential stress of PMs, (b) Tangential stress of the carbon fiber sleeve.

\section{A New Hybid Protective Measure}

Based on the above analysis, although pole gaps are filled with non-magnetic plastics, the carbon fiber retaining sleeve still has a very high bending stress and a high edge stress at high-temperature and high-speed operations, which may affect the reliability of the carbon fiber retaining sleeve. Therefore, a new hybrid protective measure is proposed, as shown in Fig. 12. The sleeve consists of both Ti-alloy and carbon fiber. The thickness of Ti-alloy and carbon fiber is set to 1 and $6 \mathrm{~mm}$, respectively. The carbon fiber bundles the Ti-alloy by specialized companies and then the hybrid retaining sleeve is mounted on the rotor. Fig. 13 shows that the hybrid retaining sleeve displacement is only $0.042 \mathrm{~mm}$ in the inner surface at $150^{\circ} \mathrm{C}$. It is difficult to fit the hybrid sleeve into the rotor in the assembly process and therefore the clearance fit is chosen for the rotor assembly. The air between the hybrid retaining sleeve and PMs is pumped out and the adhesive is filled in the clearance to enhance the integrity of the rotor.

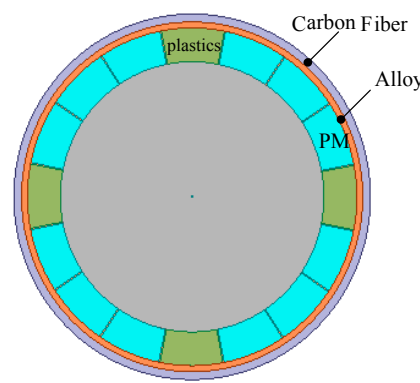

Fig. 12. The proposed hybrid protective measure.

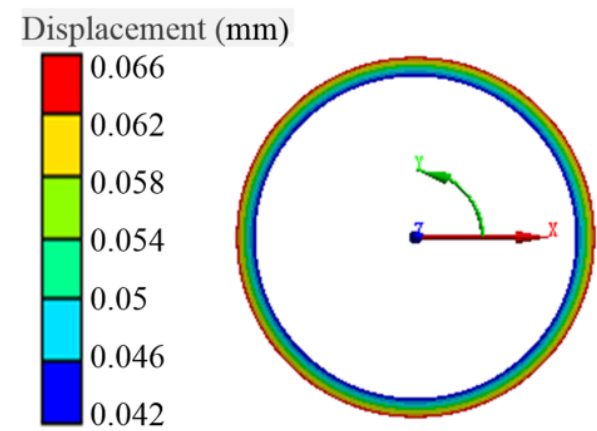

Fig.13. The hybrid retaining sleeve displacement at $150^{\circ} \mathrm{C}$.

When the machine runs at $22 \mathrm{krpm}$, the rotor stresses at $20^{\circ} \mathrm{C}$ and $150^{\circ} \mathrm{C}$ are obtained; numerical results are 
presented in Figs. 14 and 15 and summarized in Table IV. It can be observed that both the radial and tangential stresses of the PMs and sleeve at $150^{\circ} \mathrm{C}$ are much greater than these at $20^{\circ} \mathrm{C}$, with a minimal increase of $47 \%$ and a maximal increase of $376 \%$. For the hybrid protective measure, the tangential stress of the sleeve is $118 \mathrm{MPa}$ at $20^{\circ} \mathrm{C}$, and $395 \mathrm{MPa}$ at $150^{\circ} \mathrm{C}$, which are about $100 \mathrm{MPa}$ lower than these for the carbon fiber retaining sleeve. The radial stress of PMs and tangential stress of PMs with the hybrid retaining sleeve are about $5 \mathrm{MPa}$ greater than these for the carbon fiber retaining sleeve. The radial stress of PMs is the compressive stress and is also much lower than the maximum permissible compression stress of PMs (i.e. $1100 \mathrm{MPa}$ ). All rotor stresses with both retaining sleeve at $20^{\circ} \mathrm{C}$ and $150^{\circ} \mathrm{C}$ are lower than the maximum permissible stresses of materials. It proves that the hybrid protective measure can improve the rotor performance in terms of the bending stress and edge stress at high-temperature high-speed operations.
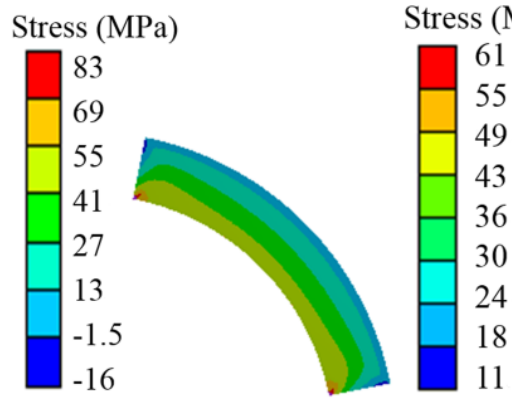

(a)

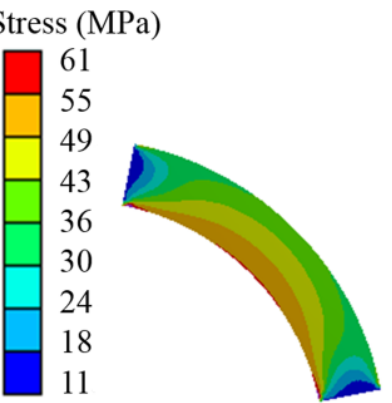

(b)

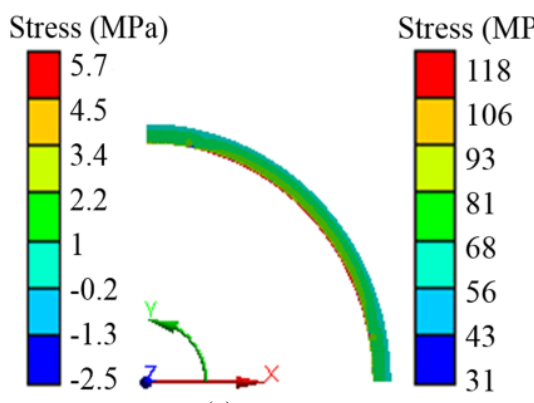

(c)

Fig. 14. The hybrid retaining sleeve stress at $22 \mathrm{krpm}$ and $20^{\circ} \mathrm{C}$. (a) Radial stress of PMs. (b) Tangential stress of PMs. (c) Radial stress of the carbon fiber sleeve. (d) Tangential stress of the carbon fiber sleeve.
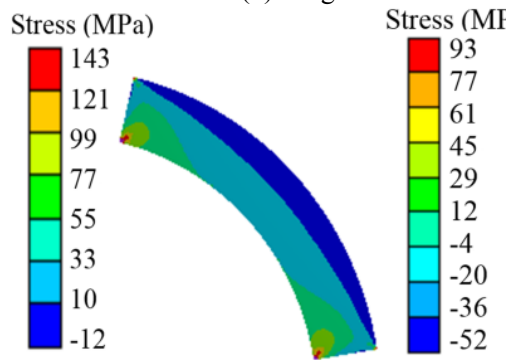

(a)

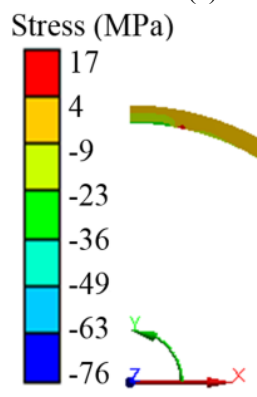

(c)

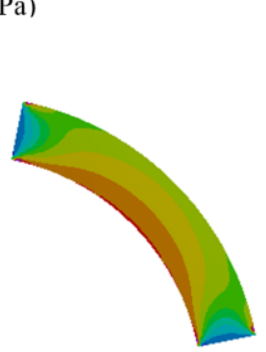

(b)

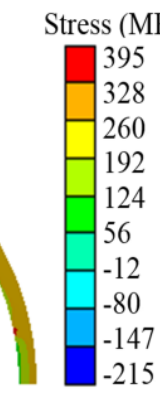

$-215$

a)
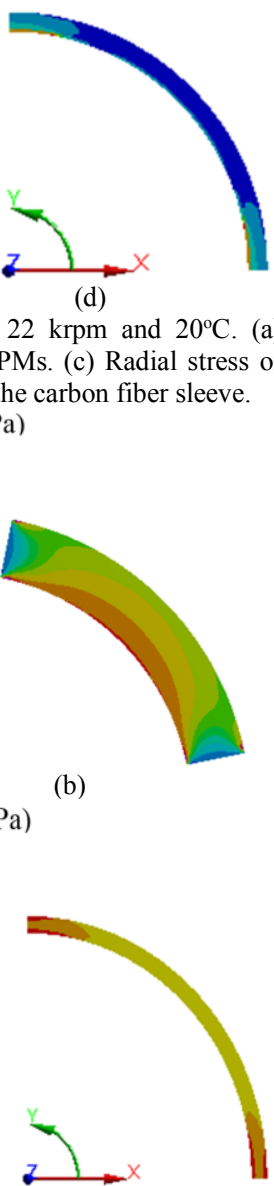

(d)
Fig. 15. The hybrid retaining sleeve stress at $22 \mathrm{krpm}$ and $150^{\circ} \mathrm{C}$. (a) Radial stress of PMs. (b) Tangential stress of PMs. (c) Radial stress of the carbon fiber sleeve. (d) Tangential stress of the carbon fiber sleeve.

TABLE IV COMPARISON OF THE ROTOR STRESS FOR BOTH SLEEVES

\begin{tabular}{|l|c|c|c|c|}
\hline \multirow{2}{*}{ Stress (MPa) } & \multicolumn{2}{|c|}{ Carbon fiber sleeve } & \multicolumn{2}{c|}{ Hybrid sleeve } \\
\cline { 2 - 5 } & $20^{\circ} \mathrm{C}$ & $150^{\circ} \mathrm{C}$ & $20^{\circ} \mathrm{C}$ & $150^{\circ} \mathrm{C}$ \\
\hline Radial stress of PMs & 78 & 140 & 83 & 143 \\
\hline Tangential stress of PMs & 49 & 88 & 61 & 93 \\
\hline Radial stress of sleeve & -17 & -81 & -2.5 & -76 \\
\hline Tangential stress of sleeve & 201 & 488 & 118 & 395 \\
\hline
\end{tabular}

\section{ROTOR LOSSES AND THERMAL ANALYSIS OF BOTH SLEEVE STRUCTURES}

For the hybrid carbon fiber sleeve, the Ti-alloy layer would have an impact on the rotor eddy current loss and rotor temperature. The rotor eddy current losses for the two sleeve structures (carbon fiber retaining sleeve and hybrid retaining sleeve) are obtained by FEM and tabulated in Table $\mathrm{V}$ for comparison. The sleeve thickness of both sleeve structures is the same. The isotropic conductivity of carbon fiber is assumed in the calculation. The carbon fiber has a low electrical conductivity, but the sleeve is quite thick $(7 \mathrm{~mm})$. The PM is only $17 \mathrm{~mm}$ thick and the air gap is $3 \mathrm{~mm}$. The eddy current loss in the carbon fiber sleeve is higher than that in the PM. The PM eddy current loss in the hybrid retaining sleeve is lower than in the carbon fiber retaining sleeve because the Ti-alloy can shield PMs partly. However, the total rotor eddy current loss in the hybrid retaining sleeve is about $7 \%$ higher than the carbon fiber retaining sleeve. Overall, the carbon fiber retaining sleeve has the lower total loss but also the highest tangential stress on the sleeve (Table IV).

\section{TABLE V}

Rotor EDDy CuRRENT LOSSES With BOTH SLEEVE STRUCTURES

\begin{tabular}{|c|c|c|}
\hline Loss (W) & Carbon Fiber Sleeve & Hybrid Sleeve \\
\hline Carbon Fiber & 1520 & 1258 \\
\hline Ti-alloy & 0 & 898 \\
\hline PM & 1040 & 590 \\
\hline Total & 2560 & 2746 \\
\hline
\end{tabular}

In order to remove the generated heat in the rotor, a cooling system is designed to utlize both air and water cooling. The machine temperatures with different sleeve structures are calculated using the fluid-solid coupling method in Ansys CFX. The thermal results with two sleeve structures are shown in Fig. 16 and the maximum temperatures for the rotor, winding and stator core are compared in Fig. 17. The rotor maximum temperature occurs in the sleeve; the winding maximum temperature and the stator maximum temperature occur near the outlet of the ventilation duct. The total rotor eddy current loss in the hybrid retaining sleeve is approximately $200 \mathrm{~W}$ higher than the carbon fiber retaining sleeve, but the maximum rotor temperature with the hybrid retaining sleeve is about $1^{\circ} \mathrm{C}$ lower than the carbon fiber retaining sleeve, because Ti-alloy has a much higher thermal conductivity than carbon fiber. The winding and stator core temperatures for both sleeve structures are almost the same. 


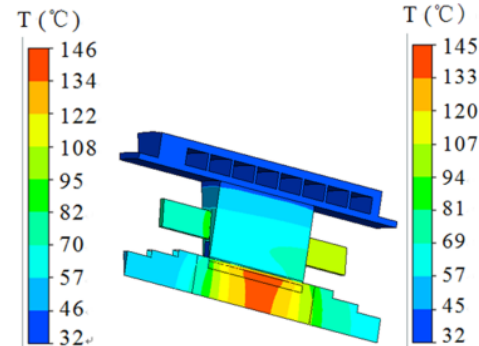

(a)

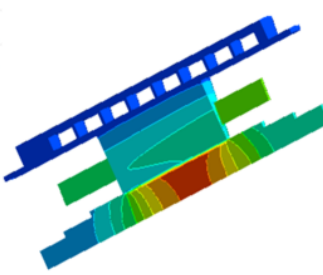

(b)
Fig. 16 Thermal results with two sleeve structures. (a) Carbon fiber retaining sleeve. (b) Hybrid retaining sleeve.

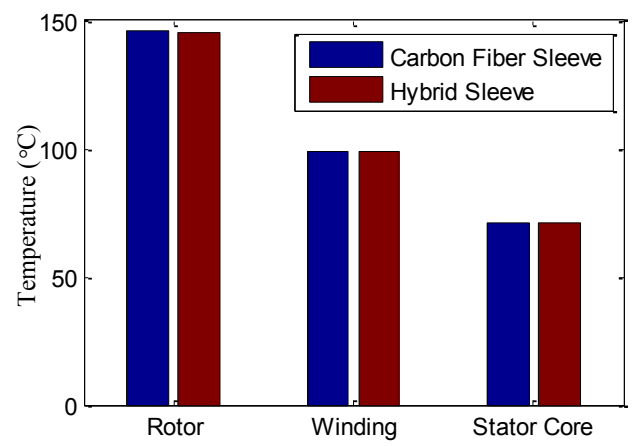

Fig. 17 Machine temperatures with two sleeve structures.

\section{PROTOTYPES AND EXPERIMENTAL VALIDATION}

From the previous analysis, the two rotors with a carbon fiber retaining sleeve and a hybrid retaining sleeve are manufactured, as shown in Fig. 18. A stress test platform is developed (see Fig. 19) to verify the reliability of rotor designs prior to installing in the stator. In the test, an inverter-fed induction motor and a high-speed gearbox are used to drive the rotor. For safety reasons, the rotors are surrounded by machine shield. Both rotors run safely at the speed of $12 \mathrm{krpm}, 18 \mathrm{krpm}, 22 \mathrm{krpm}$ steadily for hours. Afterwards, the rotors are taken out and physically examined for any damage. The health condition of the two rotors has validated the rotor stress analysis.

In addition, electromagnetic and thermal tests at the full-load condition are also carried out on the prototype (see the test rig in Fig. 20) while the rotor with the carbon fiber retaining sleeve runs up to $18 \mathrm{krpm}$.

The developed PMSM drives a MW induction generator through a gearbox. The prototype and load (an induction generator) are controlled by two inverters. The speed of the prototype and load machine can be regulated by the PWM frequency of the inverter and gearbox, respectively. In order to improve the thermal transfer within the machine, an air-water cooling system is used in the tests. The winding temperature is measured by three thermistors inserted in the windings. Fig. 21 shows the measured waveforms of the phase current at the rated condition. The rms of the current reads $235 \mathrm{~A}$, which agrees well with the simulation result. The numerical and experimental results are also listed in Table VI for comparison. In the tests, the prototype runs at $18 \mathrm{krpm}$ for 7.7 hours. The measured winding temperature is about $65^{\circ} \mathrm{C}$ while the estimated stator temperature from simulation is $69^{\circ} \mathrm{C}$. The input power and the induced line voltage measure $1.15 \mathrm{MW}$ and $3.14 \mathrm{kV}$, and the power factor is 0.96 . All results are agreeing well with numerical calculations.

Overall, it is proved that the analysis and experiments on the proposed PMSM with the rotor retaining sleeve are effective.

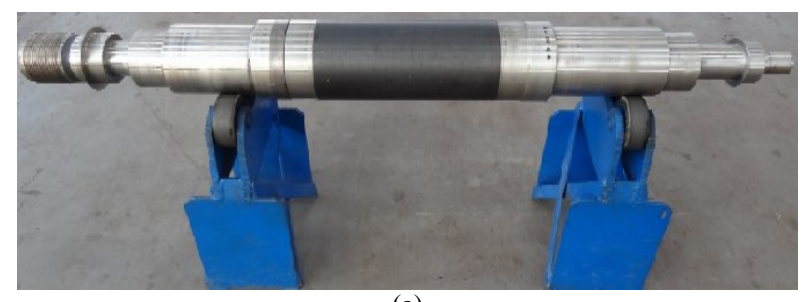

(a)

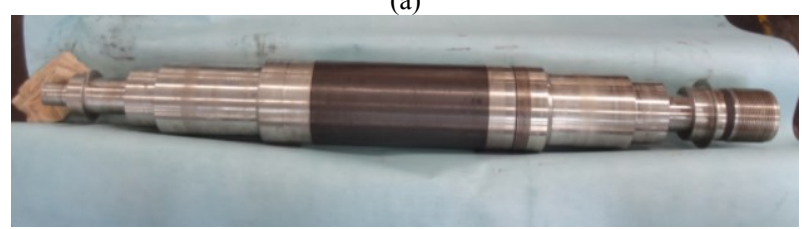

(b)

Fig. 18. Photographs of the two rotors. (a) Carbon fiber retaining sleeve. (b) Hybrid retaining sleeve.

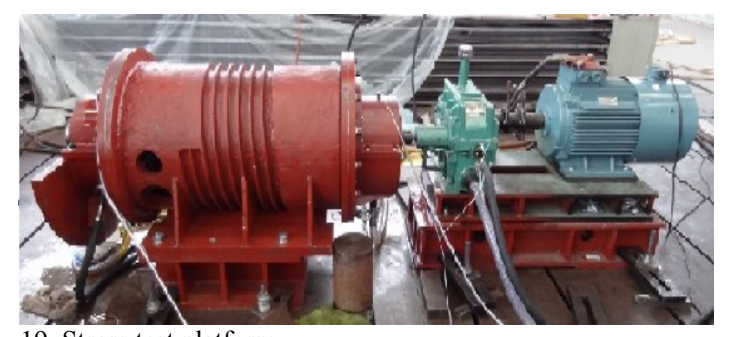

Fig. 19. Stress test platform.

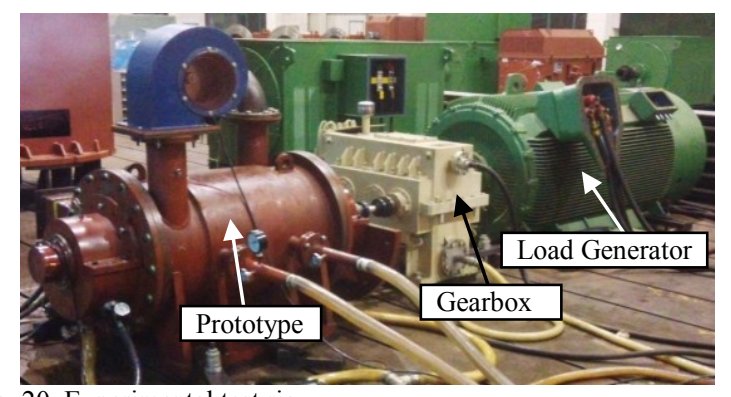

Fig. 20. Experimental test rig.

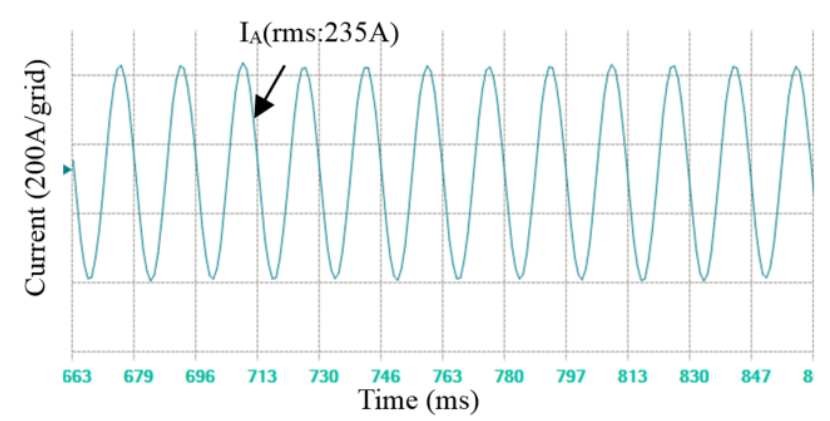

Fig. 21. Waveforms of the phase current at full load.

TABLE VI COMPARISON BETWEEN NUMERICAL CALCULATIONS AND EXPERIMENTAL MEASUREMENTS

\begin{tabular}{|c|c|c|}
\hline & Calculation & Measurement \\
\hline Runing time (hour) & N/A & 7.7 \\
\hline Speed (krpm) & 18 & 18 \\
\hline Input power $(\mathrm{MW})$ & 1.147 & 1.15 \\
\hline Induced line voltage $(\mathrm{kV})$ & 3.145 & 3.14 \\
\hline Phase current $(\mathrm{A})$ & 224 & 235 \\
\hline Power factor & 0.965 & 0.96 \\
\hline Winding temperature $\left({ }^{\circ} \mathrm{C}\right)$ & 69 & 65 \\
\hline
\end{tabular}




\section{CONCLUSION}

In this paper, a PMSM rated $1.12 \mathrm{MW}$ and $18 \mathrm{krpm}$ is investigated by the $2 \mathrm{D}$ and $3 \mathrm{D}$ FEM, and experimental tests, with a focus on the rotor retaining sleeve design. The stress of three different PM configurations and three materials in the pole filler are numerically studied for speeds up to 1.2 times the rated speed. The analytical, numerical and experimental results have suggested that it is effective to reduce the sleeve stress by filling the pole gap. In addition, the effect of the operating temperature, rotor speed, retaining sleeve thickness and interference fit are investigated and optimized. Then a new hybrid sleeve design is proposed which consists of Ti-alloy and carbon fiber. The rotor stresses at $20^{\circ} \mathrm{C}$ and $150^{\circ} \mathrm{C}$ are analyzed and compared to the carbon fiber sleeve at $22 \mathrm{krpm}$. Rotor losses and thermal analysis of both retaining sleeve structures are numerically computed. Two rotors (one with a carbon fiber retaining sleeve and the other with a hybrid retaining sleeve) are prototyped and experimentally tested at a speed of $22 \mathrm{krpm}$. Electromagnetic and thermal tests on the prototype with the rotor using a carbon fiber retaining sleeve are conducted at the rated condition. Experimental results agree well with numerical results, and confirm the effectiveness of the proposed PMSM and its retaining sleeve design.

\section{ACKNOWLEDGMENT}

The appreciation is expressed for Professor Fengxiang Wang for helpful advice in the machine design. This work was supported by Changjiang Scholars Program, Innovative Research Team in University of China (IRT1072), National Natural Science Foundation of China (51207094) and FP7 IRSES Project (318925).

\section{REFERENCES}

[1] Z. Kolondzovski, A. Arkkio, J. Larjola, and P. Sallinen, "Power limits of high-speed permanent-magnet electrical machines for compressor applications," IEEE Trans. Energy Convers., Vol. 26, No. 1, pp. 73-82, March 2011.

[2] J. J. H. Paulides, G. W. Jewell and D. Howe, "An evaluation of alternative stator lamination materials for a high-speed, $1.5 \mathrm{MW}$ permanent-magnet generator," IEEE Trans. Magn., Vol. 40, No. 4, pp. 2041-2043, Jul. 2004.

[3] O. Aglen, A. Anderson, "Thermal analysis of a high-speed generator," in Proc. 38th Ind. Appl. (IAS) Annual. Meeting, pp. 547-554, Oct. 2003.

[4] J. D. Ede, Z. Q. Zhu, and D. Howe, "Rotor resonances of high-speed permanent-magnet brushless machines," IEEE Trans. Ind. Appl., Vol. 38, No. 6, pp. 1542-1548, Nov. 2002.

[5] L. Papini, T. Raminosoa, D. Gerada, C. Gerada, "A high-speed permanent-magnet machine for fault-tolerant drivetrains," IEEE Trans. Ind. Elec., Vol. 61, No. 6, pp. 3071-3080, 2014.

[6] N. Bianchi, S. Bolognani and F. Luise, "Potentials and limits of high-speed PM motors," IEEE Trans. Ind. Appl., Vol. 40, No. 6, pp. 1570-1578, Nov/Dec. 2004.

[7] A. Borisavljevic, H. Polinder and J. A. Ferreira, "On the speed limits of permanent-magnet machines," IEEE Trans. Ind. Electron., Vol. 57, No. 1, pp. 220-227, Jan. 2010.

[8] A. Tenconi, S. Vaschetto, A. Vigliani, "Electrical machines for high-speed applications: design considerations and tradeoffs," IEEE Trans. Ind. Elec., Vol. 61, No. 6, pp. 3022-3029, 2014.

[9] D. Gerada, A. Mebarki, N. L. Brown, C. Gerada, A. Cavagnino, A Boglietti, "High-speed electrical machines: technologies, trends, and developments," IEEE Trans. Ind. Elec., Vol. 61, No. 6, pp. 2946-2959, 2014.

[10] W. L. Soong, G. B. Kliman, R. N. Johnson, R. A. White, and J. E. Miller, "Novel high-speed induction motor for a commercial centrifugal compressor," IEEE Trans. Ind. App., Vol. 36, No. 3, pp. 706-713,May 2000.
[11] J. Wang, F. Wang, W. Bao, and E. Guan, "Rotor design and strength analysis of high speed permanent magnet machine," Proceedings of the CSEE, Vol. 25, No. 15, pp. 140-145, Aug. 2005.

[12] W. Cheng, H. Geng, S. Feng, L. Yu, Y. Sun, L. Yang, "Rotor strength analysis of high-speed permanent magnet synchronous motors," Proceedings of the CSEE, Vol. 32, No. 27, pp. 87-94, Sep. 2012.

[13] J. Dong, Y. Huang, L. Jin, H. Lin, H. Yang, "Thermal optimization of a high-speed permanent magnet motor," IEEE Trans. Magn., Vol. 50, No. 2, pp. 749-752, 2014.

[14] F. Zhou, J. Shen, W. Fei and R. Lin, "Study of retaining sleeve and conductive shield and their influence on rotor loss in high-speed PM BLDC motors," IEEE Trans. Magn., Vol. 42, No. 10, pp. 3398-3400, Oct. 2006.

[15] M. Nakano, H. Kometani, and M. Kawamura, "A study on eddy-current losses in rotors of surface permanent-magnet synchronous machines," IEEE Trans. Ind. Appl., Vol. 42, No. 2, pp. 429-435, Mar./Apr. 2006.

[16] A. Binder, T. Schneider and M. Klohr, "Fixation of buried and surface-mounted magnets in high-speed permanent-magnet synchronous machines," IEEE Trans. Ind. Appl., Vol. 42, No. 4, pp. 1031-1037, July/Aug. 2006.

[17] A. Borisavljevic, H. Polinder and J.A. Ferreira, "Enclosure design for a high-speed permanent magnet rotor," IET Conference on Power Electronics, Machines and Drives (PEMD), April, 2010.

[18] J. M. Yon, P. H. Mellor, R. Wrobel, J. D. Booker and S. G. Burrow, "Analysis of semipermeable containment sleeve technology for high-speed permanent magnet machines," IEEE Trans. Energy Convers, Vol. 27, No. 3, pp. 646-653, Sept. 2012.

[19] S. S. Li, "High speed carbon fiber composite rotor's mechanics character research and energy-storage density optimization", Ph.D. dissertation, Changchun institute of optics, fine mechanics and physics, Chinese academy of science, Changchun, China, 2002. 